\title{
Randwanderung, Pendeln und Geschlecht in einer polyzentralen Region
}

\author{
Joachim Scheiner $^{1}$
}

Eingegangen: 6. Oktober 2015 / Angenommen: 22. Februar 2016 / Online publiziert: 17. März 2016

(C) Springer-Verlag Berlin Heidelberg 2016

Zusammenfassung Der Beitrag untersucht die Pendeldistanzen und die Dauer der Pendelwege im Kontext von Randwanderung und Geschlechterverhältnissen im Umland der Stadt Hagen (Westfalen). Die Ergebnisse zeigen, dass auch innerregionale Wanderungen häufig mit beruflichen Motiven verbunden sind. Die geschlechtsspezifischen Veränderungen im Pendeln nach dem Umzug sprechen für die Orientierung des Wohnstandorts am Arbeitsplatz des Mannes, wobei Frauen tendenziell die „Trailing-wife“-Position einnehmen. Darüber hinaus gehen die Wanderungen mit dem Versuch einer Begrenzung oder Verkürzung der Pendelwege auf der Haushaltsebene einher. Dies drückt sich nicht nur in der Pendeldauer aus, sondern auch in der räumlichen Konfiguration von Wohnen und Arbeiten bei erwerbstätigen Paaren, die hier über die relative Lage von Wohn- und Arbeitsorten zueinander abgebildet wurde. In einem Ring von $20-30 \mathrm{~km}$ um Hagen sind die Wohnstandortentscheidungen allerdings im Vergleich zum näheren Umland $(<20 \mathrm{~km}$ von Hagen) mit deutlich längeren Pendeldistanzen nach dem Umzug verbunden, was den klassischen Befunden der Suburbanisierungsforschung entspricht. Insgesamt sprechen die Ergebnisse für sehr komplexe Beziehungen zwischen Geschlecht, sozialem Status, Wohnstandortwahl und Pendelverhalten. Dies wird auch vor dem Hintergrund der polyzentrischen Struktur der Region interpretiert. Schlussfolgerungen für die weitere Forschung ergeben sich aus

Prof. Dr. Joachim Scheiner

joachim.scheiner@tu-dortmund.de

1 Fachgebiet Verkehrswesen und Verkehrsplanung, Fakultät Raumplanung, Technische Universität Dortmund, Dortmund, Deutschland einigen innovativen methodischen Ansätzen der Studie. Die planerische Bedeutung der Ergebnisse wird ebenfalls kurz diskutiert.

Schlüsselwörter Randwanderung · Pendeln ·

Geschlecht · Wohnstandortwahl · Polyzentrale Region

\section{Suburbanisation, Commuting and Gender in a Polycentric Region}

Abstract The paper studies commute distances and commute duration in the context of suburbanisation and gender relations in the surroundings of Hagen, Westphalia. The results show that intraregional migration is often motivated by workplace-related reasons. The genderspecific changes in commuting following the moves suggest that residential location choice tends to be driven by the male partner's workplace, while women hold a "trailing wife" position. Additionally, the moves are associated with attempts to limit or reduce commuting trip lengths on the household level. This is not only reflected in trip duration, but also in the spatial configuration of housing and work among employed couples. This is represented here by the relative location of the residence and workplaces. In a ring of $20-30 \mathrm{~km}$ around Hagen the residential choices are associated with considerably longer commuting distances, compared to municipalities located closer to Hagen $(<20 \mathrm{~km})$. This supports typical results of suburbanisation studies. Taken overall, the findings demonstrate very complex relationships between gender, social status, residential location choice and commuting. This is discussed against the backdrop of the polycentric structure of the region. Conclusions for further research can be drawn from the novel methods used in the study. The 
relevance of the results for planning is briefly discussed as well.

Keywords Suburbanisation - Commuting - Gender · Residential location choice $\cdot$ Polycentric region

\section{Einleitung}

Seit den 1960er- und 1970er-Jahren gilt es in der planungsnahen Forschung als ausgemacht, dass die Abwanderung der Haushalte aus den Städten in das Umland mit einem starken Ausmaß an Verkehrserzeugung verbunden ist. Insbesondere der Pkw-Verkehr steht dabei im Zentrum der Kritik (König 1978; Hesse 2001). Hierfür sind mehrere Argumentationslinien ausschlaggebend, die hier nur verkürzt wiedergegeben werden können.

Erstens ist die geringe Dichte und monofunktionale Flächennutzung des suburbanen Raums im Vergleich zur Stadt mit langen Pendelwegen verbunden. Zweitens sind innerregionale Wanderungen nicht durch Arbeitsplatzwechsel induziert, sondern durch Wohnbedürfnisse. Demzufolge werden Arbeitsplätze, aber auch Ausbildungsplätze und andere sozialräumliche Bindungen in den Städten beibehalten, sodass die Aktionsräume der abgewanderten Haushalte sich selbst bei einem ausreichenden wohnortnahen Arbeitsplatzangebot auf die regionale Ebene erweitern. Drittens lassen sich die entstehenden diffusen regionalen Verflechtungen nur schwer mit dem öffentlichen Personennahverkehr (ÖPNV) bedienen, sodass alltägliche Wege in der Regel mit dem Pkw zurückgelegt werden.

Diese Einschätzungen wurden nur hin und wieder in Frage gestellt, als nach den Haushalten verzögert auch Arbeitsplätze, Einzelhandel, Dienstleistungen und Freizeiteinrichtungen ihre Standorte ins Umland verlegten. Vor allem in der nordamerikanischen Stadtforschung wurde die Co-Location-Hypothese diskutiert, nach der im Zuge der Suburbanisierung von Arbeitsplätzen und Wohnen sich das Umland von den Städten funktional ablösen und zur eigenständigen Stadt mit allen dazugehörigen Funktionen ,ausreifen“ würde, was in der Folge zu einer „Re-Lokalisierung“ der funktionsräumlichen Beziehungen und entsprechend kürzeren Pendeldistanzen führen würde (Gordon/Kumar/ Richardson 1989; vgl. zur Diskussion Rahn 2011, S. 48 ff.; Guth 2014, S. 20 ff.). Stattdessen allerdings nahmen in Deutschland wie auch in anderen Ländern trotz der voranschreitenden Urbanisierung des Umlandes praktisch flächendeckend die Pendeldistanzen weiter zu (Guth 2014).

Die Debatte um Stadtentwicklung, Wohnstandortwahl und Pendeln ist verknüpft mit der Frage nach den Geschlechterspezifika dieser Prozesse. Diese intensive Diskussion wird bereits seit den späten 1970er-Jahren vor allem in der nordamerikanischen Stadtforschung geführt. Die
Literatur deutet (relativ) konsistent darauf hin, dass Frauen erstens kürzere Pendelwege zurücklegen als Männer, was in der Regel im Sinne einer Benachteiligung der Frauen interpretiert wird: als Einschränkung ihrer Mobilität, ihres Aktionsraums und des Zugangs zu Arbeitsmärkten (Hanson/ Pratt 1995; MacDonald 1999; Crane 2007).

Zweitens zeigen Studien zur Wohnstandortverlagerung von Paar- und Familienhaushalten, dass die Frauen auch beim Umzug häufig in einer benachteiligten Rolle sind (,tied mover") (Green 1997; Cooke/Boyle/Couch et al. 2009; Nisic 2010). Der Umzug bringt in der Regel einen ökonomischen Nutzen für den Gesamthaushalt, dient aber auf der Personenebene der Karriere des Mannes, während die Frau eher ökonomische Nachteile erfährt. Der Bezug zur Suburbanisierung ergibt sich aus dem Argument, dass der Mangel an Arbeitsplätzen in Verbindung mit unterentwickelten ÖPNV-Systemen im suburbanen Raum für die Frauen eine „räumliche Falle“ darstellt, in der die Optionen für einen der eigenen Qualifikation angemessenen Arbeitsplatz eingeschränkt sind.

Die gesamte bisher dargelegte Denkfigur ist allerdings mit einem monozentrischen Stadtmodell verbunden, in dem ein funktional hochentwickelter Kern von einem unterentwickelten Umland umgeben ist (,Schlafstadt“). In polyzentrischen Regionen entsteht mit der Abwanderung eines Haushalts aus einem Kern häufig Nähe zu einem oder mehreren benachbarten Kernen, sodass weder die Erwartung zunehmender Pendeldistanzen noch die Idee einer „räumlichen Falle“ für Frauen notwendigerweise zutrifft.

Dieser Beitrag untersucht anhand von Daten aus dem Umland der Stadt Hagen (Westfalen) die Pendeldistanz und Pendeldauer von Frauen und Männern vor und nach einer Wanderung aus der Kernstadt in das Umland. Dabei stehen die Fragen im Mittelpunkt, (a) ob das Geschlecht zum Verständnis des Pendelverhaltens beitragen kann und (b) zugunsten bzw. auf Kosten welchen Partners im Hinblick auf den Pendelaufwand Paare gegebenenfalls ihren Wohnstandort verlagern. Soziodemographische und räumliche Rahmenbedingungen werden über das Geschlecht hinaus ebenfalls kontrolliert, insbesondere der persönliche Beitrag beider Partner zum Haushaltseinkommen, der normalerweise in Pendler- und generell Verkehrsverhaltensstudien mangels Daten nicht berücksichtigt wird. Darüber hinaus wird - und auch dies ist in der deutschen Verkehrsforschung relativ neu - anhand der Pendeldistanzen auf Haushaltsebene sowie der Konfiguration von Wohnstandort und Arbeitsplatzstandorten in Paarhaushalten untersucht, ob die Haushalte ihren Pendelaufwand insgesamt durch den Umzug strategisch minimieren, die Wohnstandortwahl also pendeleffizient ist. Damit will der Beitrag im Sinne politischen und planerischen Grundlagenwissens zu einem besseren Verständnis der Beziehungen zwischen Pendeln, Wohnstandortwahl und Geschlecht beitragen. Gleichzeitig 
wird hiermit auch in methodischer Hinsicht teilweise Neuland betreten.

Befragt wurden 584 Haushalte mit 878 Pendlern, die in den Jahren 2011-2013 aus Hagen abgewandert sind. Hagen besitzt eine spezifische, für ein Oberzentrum untypische räumliche Lage am Rand des polyzentralen Agglomerationsraumes Ruhrgebiet. Dies führt dazu, dass ein Teil der (scheinbar) suburbanisierenden Haushalte de facto in die benachbarten Oberzentren ziehen.

Im folgenden Kapitel 2 wird als konzeptioneller Rahmen der Stand der Forschung schrittweise dargestellt: (a) zu den Beziehungen zwischen Randwanderung und Pendeln, (b) zu Geschlecht und Pendeln und schließlich (c) zu Geschlechterrollen bei Wohnstandortverlagerungen von Haushalten sowie speziell bei der Suburbanisierung. Aufgrund der Breite der Themen beschränkt sich die Literatur jeweils auf exemplarische bzw. wesentliche Quellen. Anschließend werden die Daten sowie die Analysestrategie vorgestellt (Kapitel 3). Darauf folgt in Kapitel 4 die Darstellung der Ergebnisse. Eine kurze zusammenfassende Interpretation sowie Schlussfolgerungen für die weitere Forschung und Planung (Kapitel 5) schließen den Beitrag.

\section{Stand der Forschung}

\subsection{Randwanderung und Pendeln}

Eine umfangreiche Literatur untersucht bereits seit den 1970er- und verstärkt seit den 1990er-Jahren die Zusammenhänge zwischen Raumstruktur und Verkehrsverhalten. Dabei spielen auch Pendelwege eine wichtige Rolle (vgl. etwa Zolnik 2011 für die USA; Guth 2014 für Deutschland). Bezüglich der Raumstruktur steht die Unterscheidung zwischen zentral-urbanen und eher peripher-suburbanen Umfeldern im Mittelpunkt (Boarnet 2011 für die USA; Überblick über die internationale Literatur bei Siedentop/ Hesse 2005). Die Ergebnisse dieser Studien lassen sich zu drei Kernaussagen zusammenfassen: Erstens korrespondiert eine dichte, kompakte und nutzungsgemischte Siedlungsstruktur mit relativ kurzen Wegen der Bevölkerung (Verkehrssparsamkeit) und zweitens mit einem hohen Anteil der Verkehrsmittel des Umweltverbundes (zu Fuß, Fahrrad, ÖPNV) am Gesamtverkehr. Drittens ist eine zentrumsnahe Lage im Stadtraum und in der Region (auch unabhängig von der internen Flächennutzung eines Gebiets) verbunden mit kurzen Wegen. Insgesamt korrespondieren damit urbane Strukturen mit kurzen Wegen und geringer Nutzung des Pkw, suburbane Strukturen dagegen mit langen Wegen und starker Nutzung des Pkw.

Diese Befunde bleiben trotz vielfacher Differenzierung im Detail in der Regel auch bei Kontrolle von soziodemographischen Einflüssen und Mobilitätspräferenzen (residentielle Selbstselektion, siehe Scheiner 2009; Boarnet 2011) relativ stabil oder nivellieren sich nur teilweise. Weiter differenziert werden sie durch die Einbeziehung von Wohn- und Mobilitätsbiografien. So zeigt sich, dass die langen Pendelwege im suburbanen Raum vor allem auf die aus der Kernstadt zugezogenen Haushalte zurückgehen, deren Pendelwege mit dem Umzug länger werden (Prillwitz/ Harms/Lanzendorf 2007), weil sie ihre Arbeitsplätze (und andere Bindungen) in der Kernstadt beibehalten, während die alteingesessene Bevölkerung kürzere Wege zurücklegt (Geier/Holz-Rau/Krafft-Neuhäuser 2001; Scheiner 2009, S. 162 ff.). Die Randwanderung geht demnach mit einer Verlängerung der Pendelwege einher.

\subsection{Pendeln von Männern und Frauen}

Auch die Debatte um Geschlechterunterschiede im Pendeln reicht bis in die 1970er-Jahre zurück. Weltweit weitgehend konsistent zeigt diese Forschung, dass Frauen kürzere Pendeldistanzen zurücklegen und für das Pendeln weniger Zeit aufwenden als Männer (Crane 2007 für die USA; Hjorthol/Vagane 2014 für Norwegen; SurprenantLegault/Patterson/El-Geneidy 2013 für Montreal, Kanada; McQuaid/Chen 2012 für das UK; Konrad 2015 für Deutschland) sowie weniger häufig fernpendeln (Schneider/ Limmer/Ruckdeschel 2002, S. 79 und 145 für Deutschland). Im Wesentlichen lassen sich die Hypothesen zur Erklärung dieser Befunde in fünf Punkten zusammenfassen (zur Diskussion siehe Hanson/Pratt 1995; Camstra 1996; MacDonald 1999; Clark/Wang 2005; Crane 2007; Scheiner/ Holz-Rau 2012).

1. Ökonomische Macht und Arbeitsmarkt: Geringere berufliche Qualifikation und ein geringeres Einkommen auch bei gleicher Qualifikation führen zu geringerer ökonomischer Macht der Frauen, was deren Aktionsradien einschränkt. Suburbane Umfelder mit eingeschränktem Zugang zu Arbeitsmärkten werden teilweise als regelrechte ,räumliche Falle“ (,spatial entrapment") beschrieben (MacDonald 1999). Zudem ist die Segregation von Arbeitsplätzen in ,typischen Frauenjobs“ geringer (MacDonald 1999). Auch wenn es sich hier vorwiegend um eine nordamerikanische Debatte der 1990er-Jahre handelt, spielen diese Hypothesen auch heute und in Europa eine wichtige Rolle (Hudson 2014). Allerdings ist der theoretische Zusammenhang zwischen ökonomischer Macht und Pendeldistanz keineswegs eindeutig. Plausibel wäre auch, dass die Person mit dem höchsten Beitrag zum Haushaltseinkommen den Wohnort bestimmt. Demnach ginge mit ökonomischer Macht eine kürzere Pendeldistanz einher (Shearmur 2006). 
2. Zugang zu Ressourcen: In einer breiteren Perspektive spielen neben Geld auch andere Ressourcen eine wichtige Rolle, zu denen Frauen möglicherweise keinen ausreichenden Zugang haben. Hierzu zählen Zeit, Pkw-Verfügbarkeit bzw. generell Mobilität, Kinderbetreuungsleistungen und persönliche Unterstützungsnetzwerke (Rahn 2011; Hudson 2014).

3. Soziale Rollen: Frauen übernehmen in Paarhaushalten in der Regel den größeren Anteil an Haushalts- und Familienarbeit. Dies schränkt ihre ökonomische Unabhängigkeit, ihre Aktionsräume und ihren Zugang zu Arbeitsmärkten ein, was letztlich zu kürzeren Pendelwegen führt (McQuaid/Chen 2012). Besonders ausgeprägt gilt dies für Haushalte mit Kindern (Konrad 2015).

4. Präferenzen:Aus sozialpsychologischer Perspektive wird hin und wieder der Einfluss von Gendereinstellungen, Normen und Präferenzen untersucht (Hakim 2000). Hierzu zählen im Verkehrskontext die möglicherweise stärkeren ökologischen Normen, geringer ausgeprägte Routinen und stärkere Veränderungsbereitschaft von Frauen (Matthies/Kuhn/Klöckner 2002; Hjorthol 2008).

5. Patriarchat: Nach dieser Hypothese beherrscht das Patriarchat als hierarchisches System sowohl ökonomische Ungleichheiten als auch andere Ressourcen, soziale Rollen, Normen und Präferenzen (Walby 1990). Demnach wären Geschlechterunterschiede im (Pendel-) Verhalten letztlich Ausdruck patriarchaler Machtverhältnisse, selbst wenn sie auf scheinbar frei getroffenen individuellen Entscheidungen basieren.

Die kürzeren Pendeldistanzen von Frauen als Output dieser Hypothesen werden in der Regel als Benachteiligung gedeutet. Die Schwierigkeiten der Interpretation zeigen sich, wenn in einigen Studien besonders lange Pendelwege von Frauen wiederum - mit jeweils guten Gründen - als Benachteiligung interpretiert werden (MacDonald 1999 und Clark/Wang 2005 für die USA; Kawase 2004 für Japan). Die Interpretation langer Wege als Benachteiligung steht in Übereinstimmung mit der klassischen verkehrswissenschaftlichen Theorie, wonach der Distanz- und Zeitaufwand für Wege einen Kostenfaktor („disutility“) darstellt. Die umgekehrte Interpretation kurzer Wege als Benachteiligung in der feministischen (und weiteren genderorientierten) Verkehrsforschung steht dagegen im Gegensatz zur klassischen - geschlechtsneutralen - Verkehrsökonomie (vgl. Gordon/ Kumar/Richardson 1989 für eine kritische Gegenposition).

\subsection{Wohnstandortverlagerung von Paaren, Pendeln und Geschlecht}

Die Wohnstandortverlagerung und damit auch die Randwanderung von Haushalten folgt im Sinne der
Humankapitaltheorie ökonomisch dem Prinzip der Nutzenmaximierung: Ein Haushalt nimmt Transaktionskosten für das Wandern auf sich, um am neuen Wohnort einen möglichst hohen Nutzen zu erzielen. In dieser neoklassischen Haushaltslogik ist kein Raum für eine eigenständige Rolle des Geschlechts, weil es für den Haushalt ökonomisch keine Rolle spielt, welcher Partner den Nutzen für den Haushalt erwirtschaftet (Becker 1981).

Eine seit den 1970er-Jahren entwickelte geschlechterrollentheoretische Perspektive (Duncan/Perrucci 1976) behauptet demgegenüber, dass in der Wohnstandortentscheidung auch Geschlechternormen über die Zuständigkeit für bestimmte Rollen (Einkommen generieren, Kinder erziehen, den Haushalt führen) von Bedeutung sind und dass Paare in der Wohnstandortverlagerung diese Rollen berücksichtigen. Da dem Mann die Rolle des „Brötchenverdieners" zukommt, folgt der Wohnstandort dem Arbeitsplatz des Mannes, während die Frau als ,Anhängsel“ (,trailing wife“, „tied spouse“ oder „tied mover") mit umzieht. Dies gilt besonders stark für Haushalte mit Kindern, in denen die Frauen besonders stark die Verantwortung für Haushaltsund Familienarbeit übernehmen.

Für diese These gibt es zahlreiche Belege. Erstens erweisen sich in der Erfragung von Umzugsgründen von Paaren mehrheitlich die Männer und ihre Karriere als treibende Kräfte des Umzugs, während die Mehrheit der „tied mover“ frustriert ist über die erbrachten Opfer des Umzugs (Green 1997 für hochqualifizierte Paare im UK). Zweitens lässt sich aus der ökonomischen Macht zweier Partner nach dem Umzug (gemessen über die Erwerbsbeteiligung oder die Einkommensverteilung im Haushalt) sowie aus deren Veränderung nach dem Umzug schließen, dass der Umzug häufig der Karriere des Mannes dient, während die Frau individuell eher ökonomische Nachteile erfährt, auch wenn sie in ihrer Rolle als Mitglied des Haushalts durchaus davon profitieren kann (Cooke/Boyle/Couch et al. 2009 für die USA und das UK; Camstra 1996 für die Niederlande; Nisic 2010 für Deutschland).

Die Randwanderung von Haushalten spielt in dieser Forschung eine spezifische Rolle. Der suburbane Raum gilt als Ort traditioneller Geschlechterbeziehungen und damit im Zuge der Modernisierung des Geschlechterverhältnisses als Auslaufmodell (zur Diskussion vgl. Rahn 2011, S. 16 ff.). Die Gründung einer Familie stellt häufig den Auslöser der Randwanderung dar (McQuaid/Chen 2012, S. 68) und ist mit einer „Re-Traditionalisierung“ der Geschlechterverhältnisse in Partnerschaften verbunden (Grunow/Schulz/ Blossfeld 2012). Gentrifizierte innerstädtische Gebiete der Mittelschicht gelten dagegen als Orte neuer Modelle, vor allem bezüglich der Erwerbsbeteiligung von Frauen (Frank 2011). So finden Hjorthol und Vagane (2014) in Schweden, dass Frauen in Großstädten häufiger Vollzeit erwerbstätig sind als Frauen im Umland; sie interpretieren dies als 
Benachteiligung der Frauen im Umland (vgl. auch Frank 2011).

In Pendlerstudien spielt diese Forschung seit Langem eine wichtige Rolle (Rahn 2011, S. 46 ff.). Die Randwanderung von Haushalten führt tendenziell zu längeren Pendeldistanzen, weil sie normalerweise den Wohnpräferenzen des Haushalts oder dem Wohnungsmarkt folgt, während der Arbeitsplatz in der Kernstadt beibehalten wird (vgl. Kapitel 2.1). Dagegen sind Umzüge über längere Distanz häufig durch den Arbeitsplatz induziert und führen damit eher zu kürzeren Pendeldistanzen (Camstra 1996). Dies legt nahe, dass nicht nur die Umzugsdistanz, sondern auch das Umzugsmotiv in Pendelstudien zu berücksichtigen sind. Bei einer Wanderung, die dem Arbeitsplatz des Mannes folgt, ist nach der „Tied-mover"-Hypothese zu erwarten, dass Frauen, die vor dem Umzug bereits erwerbstätig sind, nach dem Umzug besonders lange Pendelwege aufweisen, sofern sie den Arbeitsplatz beibehalten (Camstra 1996). Wenn die Frau den Arbeitsplatz wechselt, ist dagegen nach der „Spatial-entrapment“-Hypothese zu erwarten, dass sie sich einen neuen - möglicherweise minderwertige(re)n Arbeitsplatz in der Nähe des neuen Wohnortes sucht. Dann sollte ihr Pendelweg kürzer sein als der des Mannes.

Von zentraler Bedeutung ist in diesen Überlegungen auch die Unterscheidung zwischen Haushalten mit einer bzw. zwei Erwerbstätigen. Singles und Haushalte mit nur einer erwerbstätigen Person müssen nicht zwei Arbeitsplätze koordinieren. Dies ermöglicht kürzere Pendelwege, obwohl die empirischen Befunde keineswegs in eine eindeutige Richtung weisen (Sultana 2005; Surprenant-Legault/ Patterson/El-Geneidy 2013).

\subsection{Spezifika polyzentrischer Regionen}

In polyzentrischen Regionen stellt sich die Abwanderung aus einer Kernstadt in ihr Umland auf spezifische Weise dar. Anders als in monozentrischen Regionen führt sie nicht notwendigerweise in eine „echte“ Umlandgemeinde, sondern kann auch in ein benachbartes hochrangiges Zentrum führen.

Die Interpretation der kurzen Pendelwege von Frauen vor dem Hintergrund eines eingeschränkten Zugangs zu Arbeitsmärkten im suburbanen Raum (unterentwickelter ÖPNV, ,räumliche Falle“) ist - wie bereits beschrieben verbunden mit einem monozentrischen Stadtmodell, in dem ein funktional hochentwickelter, komplexer Kern von einem funktional unterentwickelten Umland umgeben ist, das im Wesentlichen als „Schlafstadt“" von der Kernstadt abhängig ist. Dieses Stadtmodell ist bereits in der Chicagoer Stadtsoziologie als realitätsfern kritisiert worden (Harris/Ullman 1945). Auch in monozentrischen Stadtregionen sind im Zuge der Suburbanisierung nahezu aller städtischer Funktionen (Wohnen, Freizeit, Einkauf, Bildung, Unternehmens-Head- quarters, Verwaltungen, Verkehrsknoten) neue suburbane Zentren entstanden, die einen Trend zur polyzentrischen Region nahelegen (etwa Frankfurt-Eschborn, MünchenErding). In traditionell polyzentrischen Regionen (etwa dem Ruhrgebiet) ist es umso fraglicher, ob die oben dargestellten Überlegungen je in dieser Form zutreffend waren, weil mit der Abwanderung eines Haushalts aus einem Kern häufig Nähe zu einem oder mehreren benachbarten Kernen hergestellt wird. Kurz gesagt: Polyzentrische Regionen bieten räumlich-ökonomische Optionen, die in monozentrischen Räumen nicht im gleichen Maße gegeben sind (Kloosterman/Musterd 2001). Demnach sind dort nach (innerregionalen) Abwanderungen aus einer Kernstadt weder zunehmende Pendeldistanzen noch eine ,räumliche Falle" für Frauen notwendigerweise zu erwarten.

\section{Daten und Methodik}

\subsection{Daten}

Die hier verwendeten Daten wurden im Rahmen einer Masterarbeit (Schmidt 2014) in Zusammenarbeit mit der Stadt Hagen und der Technischen Universität Dortmund, Fachgebiet Verkehrswesen und Verkehrsplanung, erhoben. Primäres Ziel war die Erarbeitung von Empfehlungen für die Hagener Wohnungspolitik. Die Bruttostichprobe wurde aus der Meldestatistik generiert. Für die Ziehung mussten zunächst Haushalte generiert werden, wobei vereinfachend aus Hagen abgewanderte Personen mit gleichem Nachnamen und gleicher Zieladresse als Haushalt betrachtet wurden. Die Bruttostichprobe entspricht der so definierten Grundgesamtheit von 4.941 Haushalten, die im Zeitraum 2011 bis 2013 aus der Stadt Hagen in das Umland abgewandert sind. Von den $4.941 \mathrm{im}$ August 2014 versandten Briefen waren 1.279 nicht zustellbar (falsche Adressangabe des Haushalts, bereits wieder verzogen, Annahme verweigert). Nach einem einmaligen Erinnerungsschreiben wurde ein Rücklauf von 584 Haushalten (15,9\% der gültigen Adressen) mit Informationen zu 878 Personen mit Angaben zum Pendeln erreicht. Diese umfassen mit oder ohne Partner sowie mit oder ohne Kinder lebende Personen. Die Ausschöpfungsquote erscheint angesichts der begrenzten Kapazitäten zur Betreuung der kontaktierten Haushalte sowie vermutlich auch deren eingeschränkter Motivation - immerhin waren sie aus Hagen abgewandert - zufriedenstellend, spiegelt aber auch den generellen Trend abnehmender Ausschöpfungsquoten wider (z. B. bei Kontiv/Mobilität ${ }^{1}$ in Deutschland von 72 auf 21\% im Zeitraum 1976-2008; Scheiner 2009, S. 77 und Infas/DLR 2010, S. 24). Dennoch ist positiv

\footnotetext{
${ }^{1}$ Kontinuierliche Erhebungen zum Verkehrsverhalten.
} 
hervorzuheben, dass mit fast $12 \%$ ein hoher Anteil der Grundgesamtheit befragt wurde.

Die Haushalte wurden schriftlich kontaktiert; erhoben wurden personen- und haushaltsbezogene Daten einschließlich des Pendelverhaltens beider Partner (soweit vorhanden) in einem Haushalt vor und nach dem Umzug. Besonderheiten der Daten sind erstens die Möglichkeit zu Vergleichen des Pendelverhaltens vor und nach dem Umzug. Zweitens wurde auch die Verteilung des Haushaltseinkommens auf beide Partner erfragt, sodass genderbezogene Fragestellungen in Bezug zur ökonomischen Verhandlungsmacht untersucht werden konnten.

\subsection{Untersuchungsraum}

Die Stadt Hagen liegt am Rand der hochverdichteten Metropolregion Rhein-Ruhr im Grenzbereich zwischen Ruhrgebiet, Sauerland und Bergischem Land. Das Umland wurde relativ großzügig definiert und enthält alle 67 Gemeinden, deren Flächenmittelpunkt maximal $40 \mathrm{~km}$ vom Zentrum Hagens entfernt ist. Dies geht bewusst über den Pendelverflechtungsbereich Hagens hinaus (Blotevogel/Münter/Terfrüchte 2009, S. 95 ff., 107 ff., 128), um Wanderungen in die weitere Region nicht auszuschließen, bleibt aber aufgrund der Nähe zu anderen, häufig dominierenden Kernen unter der in anderen Pendlerstudien angelegten Grenze für intraregionale Verflechtungen von $60 \mathrm{~km}$ Luftlinie um eine Kernstadt (Guth 2014, S. 66). Das gewählte Gebiet reicht im Norden und Westen über die benachbarten Oberzentren Dortmund, Bochum und Wuppertal hinaus. Im Süden und Osten reicht das Gebiet weit in das Sauerland und Bergische Land in die Einzugsbereiche von Köln und Siegen hinein.

Der südliche und östliche Bereich des Untersuchungsraumes besitzt deutlich suburbanen bis ländlichen Charakter. Der Untersuchungsraum ist Teil einer polyzentralen, altindustriellen Region, die heute demographisch von Bevölkerungsverlusten geprägt ist. In den nordwestlich gelegenen Ruhrgebietsstädten herrscht relativ hohe Arbeitslosigkeit (Dortmund 12,3\%, Bochum 9,8\%, Herne 12,9\%, Hagen 10,2\%). Im südöstlich gelegenen Sauerland ist die Arbeitslosigkeit deutlich geringer (je nach Kreis 4,2 bis 6,4\%; alle Zahlen: Stand September 2015²). Die Wirtschaft ist dort durch kleinere und mittlere produzierende und handwerkliche Unternehmen mit hohem Spezialisierungsgrad geprägt, die zum Teil als Weltmarktführer gelten können (Schirbaum 2014 zur sektoralen Struktur der Arbeitsplätze). Dennoch ist auch das Sauerland von demographischer Alterung, Bevölkerungsrückgang und Abwanderung geprägt (Krajewski/Werring 2013). In Bezug auf Mobilität ist die Region durch enge Pendelverflechtungen gekenn-

\footnotetext{
${ }^{2}$ Siehe https://statistik.arbeitsagentur.de (6.10.2015).
}

zeichnet (Guth 2014), vor allem im Norden und Westen, während das Sauerland einen vergleichsweise autonomen Arbeitsmarkt bildet (Blotevogel/Münter/Terfrüchte 2009, S. $107 \mathrm{ff}$.).

Hagen selbst hat in den zwei Jahrzehnten von 1993 bis 2012 nahezu 30.000 Einwohner verloren; die Bevölkerungszahl ist damit von rund 215.000 auf 185.000 zurückgegangen. Der Verlust von 17.000 Einwohnern geht dabei auf Wanderungen zurück (eigene Berechnungen auf der Grundlage der Landesdatenbank NRW ${ }^{3}$ ). Besonders negativ ist der Wanderungssaldo in den höheren Altersklassen, während bei Kindern, jungen Erwachsenen und der mittleren Altersklasse bis unter 50 Jahren die Salden relativ ausgeglichen sind.

Damit ist Hagen weder mit den klassischen Mustern der Suburbanisierung (Abwandern der Familien, Verbleiben der Älteren) noch mit dem klassischen Muster der Bildungswanderung konfrontiert (in die Städte strömende junge Erwachsene). Dies ist mit der besonderen Struktur und Lage Hagens erklärbar. Hagen profitiert nicht von starker Bildungszuwanderung, da die Fernuniversität als größte Bildungseinrichtung für Studierende keinen Wohnsitz vor Ort erfordert, leidet aber als Großstadt mit vielfältigen Ausbildungsangeboten und der Nähe zu weiteren Oberzentren auch nicht substanziell unter Bildungsabwanderung. In Bezug auf Familienwanderungen ist Hagen nicht in dem Maße wie andere Großstädte Abwanderungen ausgesetzt, da seine Siedlungsstruktur weniger von einem dominierenden, pulsierenden Stadtzentrum und urbaner Dichte geprägt, sondern sich eher ,intraurban-polyzentrisch“ (Kloosterman/ Musterd 2001) mit starken, kleinstädtisch anmutenden Subzentren darstellt. Zudem zwingt das sehr moderate Immobilienpreisniveau (LEG Immobilien AG 2014) kaum zur Abwanderung.

\subsection{Variablen}

Gegenstand dieses Beitrags ist die Pendeleffizienz von Wohnstandortentscheidungen mit dem Fokus auf der Rolle von Geschlechterunterschieden in diesem Kontext. Untersucht werden die Pendeldauer und Pendeldistanz (einfacher Weg) vor und nach einer Randwanderung auf der Individualebene. In Haushalten mit zwei erwerbstätigen Partnern wird auch in einer genderrelationalen Perspektive die Differenz zwischen den individuellen Distanzen beider Partner auf Einflüsse des Geschlechts und weiterer Variablen untersucht.

Weitere Zielvariablen beziehen sich auf die Pendeleffizienz der Wohnstandortwahl auf Haushaltsebene und sind in Studien zu Pendeln und Geschlecht eher ungewöhnlich. So wird auch die (aggregierte) Pendeldistanz auf der

\footnotetext{
${ }^{3}$ Siehe https://www.landesdatenbank.nrw.de/ldbnrw/online (01.08.2015).
} 
Haushaltsebene untersucht. In Paarhaushalten ist dies die Summe der Pendeldistanzen zweier Partner. Datenbedingt werden dabei mögliche weitere erwerbstätige Haushaltsmitglieder vernachlässigt. Die vermutlich bisher am wenigsten untersuchte Variable ist der Winkel, der durch den Wohnort als Scheitelpunkt und die Arbeits- bzw. Ausbildungsplätze (im Folgenden kurz: Arbeitsplätze) zweier Partner aufgespannt wird (Abb. 1) (Surprenant-Legault/Patterson/ El-Geneidy 2013). Liegt der Wohnstandort nahe an einer Geraden, die beide Arbeitsplätze verbindet, so resultiert dies in einem stumpfen Winkel (nahe $180^{\circ}$ ). Pendeln dagegen beide Partner nahezu in die gleiche Himmelsrichtung zu ihren Arbeitsplätzen, resultiert dies in einem spitzen Winkel (nahe $0^{\circ}$ ). In diesem Fall könnte der Haushalt durch eine Wohnstandortverlagerung eine höhere Pendeleffizienz erreichen. Wenn die Wanderung des Haushalts sich an der Lage der Arbeitsplätze orientiert, müsste sich dieser Winkel also durch den Umzug vergrößern. Es ist zu beachten, dass der Winkel allein (ohne Berücksichtigung der Wegelängen) nur bei fest gegebenen Arbeitsorten ein Effizienzmaß sein kann, da er nichts über die Pendeldistanz und -dauer sagt. Die Pendeleffizienz auf Haushaltsebene ist naturgemäß auch kein Maß der Fairness in der Geschlechterverteilung des Pendelns. Diese wird durch die individuellen Pendelwege und deren Veränderung nach dem Umzug sowie das Verhältnis der Pendelwegelängen zwischen den Partnern abgebildet.

Da die Lage der Arbeitsplätze und des Wohnorts nur auf der Postleitzahl-(PLZ-)Ebene vorliegt, kann dieser Winkel nur für Haushalte berechnet werden, bei denen Arbeitsplätze und Wohnort in drei verschiedenen PLZ-Gebieten liegen. Zusätzlich wird deshalb untersucht, in wie vielen Haushalten vor und nach dem Umzug Wohnort und mindestens ein Arbeitsplatz im gleichen PLZ-Gebiet liegen. Auch die Zunahme dieser Haushalte spräche für das Bemühen um pendeleffiziente Wohnstandortwahl.

Als Einflussfaktoren des Pendelns werden unter Bezug auf die theoretischen Überlegungen in Kapitel 2 die folgenden Größen berücksichtigt: das Geschlecht, das persönliche Einkommen und das Bildungsniveau (ökonomische Macht, Ressourcen), die Rolle beruflicher Umzugsmotive bzw. eines Arbeitsplatzwechsels, die Haushaltsstruktur (mit bzw. ohne Partner bzw. Kinder), die
Wanderungsdistanz, das räumliche Umfeld (am Zielort des Umzugs und am Ort des Arbeitsplatzes). In den personenbezogenen Analysen werden teilweise auch Variablen zur Beschreibung des Partners verwendet (Bildung, berufliche Position, Siedlungsdichte am Arbeitsort), um den Relationen und Interaktionen in Partnerschaften Rechnung zu tragen (siehe Tabellen im Anhang für eine Variablenübersicht). Die jeweiligen Spezifikationen unterscheiden sich je nach Modell.

Bezüglich des Einkommensniveaus wird auch das quadrierte Einkommen kontrolliert, um nichtlinearen Effekten Rechnung zu tragen (Shearmur 2006, S. 341). Darüber hinaus wurden das Haushaltseinkommen und der anteilige Beitrag der befragten Person zum Haushaltseinkommen (statt des absoluten Niveaus des persönlichen Einkommens) getestet, aber im Lauf des Modellierungsprozesses aufgrund fehlender Signifikanz und schwacher Effektstärken ausgeschlossen. Weitere getestete Variablen wurden aus dem gleichen Grund verworfen: Wohnungsgröße und Wohnstatus als haushaltsbezogene Statusindikatoren; der Umfang der Erwerbstätigkeit (Vollzeit, Teilzeit, Nebenjob/geringfügig/Sonstiges); die Interaktion zwischen Geschlecht und Haushaltstyp (mit/ohne Kinder); die Altersklassen der Kinder im Haushalt. Die fehlenden Einflüsse der Interaktion zwischen Geschlecht und Haushaltstyp sowie die generell schwachen Einflüsse von Kindern im Haushalt sind bemerkenswert, weil sich ein wichtiger Teil der Forschung zum Thema auf die Verantwortung von Frauen für die Familienarbeit beruft.

Der Zielort des Umzugs wird abgebildet durch die Entfernung nach Hagen sowie mit Blick darauf, ob die Wanderung in ein hochrangiges Zentrum oder in eine kleinere Gemeinde führt. Daneben wird das räumliche Umfeld am Wohnort und am Arbeitsort (wo sinnvoll, auch des Partners) durch die Siedlungsdichte abgebildet. Hierzu wurden den Befragungsdaten CORINE-Landnutzungsdaten auf der PLZ-Ebene zugespielt (siehe hierzu Einig/Jonas/ Zaspel 2009). Diese werden durch Fernerkundung erhoben. Sie produzieren (zumindest auf Kreis- und Gemeindeebene) ähnliche Ergebnisse wie amtliche Flächennutzungsdaten, lassen sich aber kleinräumlich auflösen, während amtliche Daten unterhalb der Gemeindeebene kaum erhältlich sind. Damit sind alternative Datenquellen trotz ihrer Unzuläng-
Abb. 1 Beispiele für Winkel in der Wohnort-ArbeitsplätzeKonfiguration von Paaren (Quelle: nach SurprenantLegault/Patterson/El-Geneidy 2013)

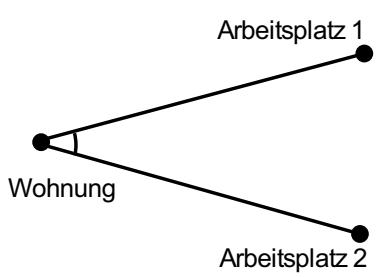

Winkel $=30^{\circ}-$ Partner pendeln in ähnliche Richtung

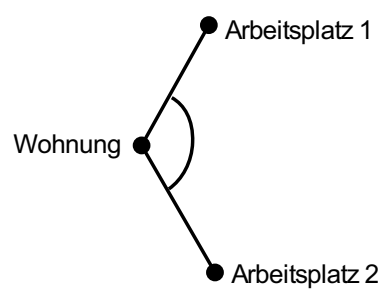

Winkel $=120^{\circ}-$ Partner pendeln in unterschiedliche Richtungen

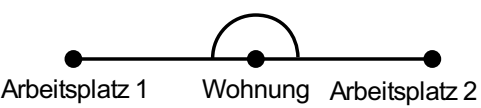

Winkel $=180^{\circ}-$ Partner pendeln in entgegengesetzte Richtungen 
lichkeiten (z. B. ist keine genauere Aussage über die Art der Flächennutzung möglich) unverzichtbar (Einig/Jonas/ Zaspel 2009). Verwendet wird hier der Anteil der Siedlungsund Verkehrsfläche an der Gesamtfläche als Indikator der Siedlungsdichte.

\subsection{Analysemethoden}

Die Ergebnisdarstellung umfasst deskriptive Analysen von Umzugsmotiven, Konfigurationen von Wohn- und Arbeitsorten, Pendeldistanzen und Pendeldauer. Darauf folgen lineare Regressionsmodelle der Pendeldistanzen (Individualebene, Haushaltsebene, Differenz zwischen Partnern). Das Signifikanzniveau beträgt $p=0.05$. In einigen Fällen werden aufgrund der teilweise kleinen Stichproben auch nicht signifikante Effekte interpretiert, wenn dies inhaltlich sinnvoll erscheint. Dies wird jeweils ausdrücklich im Text vermerkt. Die Tabellen im Anhang zeigen deskriptive Statistiken der in den Regressionsanalysen verwendeten metrischen und kategorialen Variablen sowie Referenzkategorien.

Während der Modellierung wurden ausgedehnte Versuche zur Verbesserung der Modellgütewerte unternommen. So sind die individuellen Pendeldistanzen ausgeprägt rechtsschief verteilt $(v=4,4)$. Dennoch führte weder die Logarithmierung noch der Ausschluss von Extremwerten (Pendelwege $>60 \mathrm{~km}$ ) zu deutlich besseren Gütewerten; teils wurden diese noch schlechter. Zudem wurden probeweise Ausbildungswege und Berufswege separat analysiert. Auch dies führte nicht zu besserer Modellgüte. In die Analysen gehen also sowohl Ausbildungs- als auch Berufswege ein.

\section{Ergebnisse}

Die Ergebnisdarstellung ist wie folgt aufgebaut: Auf eine Übersicht (4.1) der Bedeutung von beruflichen Umzugsmotiven und Arbeitsplatzwechseln folgen (4.2) deskriptive Analysen des Pendelns vor und nach dem Umzug sowie der entsprechenden Veränderungen im Vergleich zwischen den Geschlechtern und zwischen Haushaltstypen. Darauf
(4.3) folgen ebenfalls deskriptive Analysen zur Rolle des Arbeitsplatzwechsels bei Frauen für das Pendeln. Dies lässt Schlussfolgerungen $\mathrm{zu}$ möglichen eingeschränkten Suchradien (,räumliche Falle“) zu. Die deskriptiven Darstellungen werden beschlossen (4.4) mit einer Vorstellung der Konfiguration von Wohnort und Arbeitsorten auf der Haushaltsebene bei Paarhaushalten. Darauf folgt eine Serie von Regressionsmodellen zu Einflussfaktoren (4.5) der individuellen Pendeldistanz am neuen Wohnstandort mit Blick auf die Rolle des Geschlechts und anderer Faktoren, (4.6) der Haushalts-Pendeldistanz als Indikator der Pendeleffizienz der Wohnstandortwahl auf der Haushaltsebene, und (4.7) der Pendeldifferenz in Partnerschaften als Ausdruck der Geschlechterrelation.

\subsection{Umzugsmotive: Berufliche Wanderungen in der Region und Arbeitsplatzwechsel}

Die häufigsten Umzugsmotive sind familiäre und persönliche Gründe, wie es bei innerregionalen Wanderungen zu erwarten ist (Tabelle 1). Bei immerhin 23,6\% der Befragten sind berufliche oder ausbildungsbezogene Gründe ausschlaggebend. Bei weiteren 23,1\% treten diese zumindest als Nebenmotiv auf. Damit spielen bei fast der Hälfte der Befragten berufliche Motive eine Rolle. Dies entspricht Ergebnissen aus der Region Köln, wo 45\% der Befragten, die einen innerregionalen Umzug planen (ohne Umzüge innerhalb der eigenen Wohngemeinde), berufliche Motive angaben (Kasper/Scheiner 2006, S. 180).

Von den Befragten mit beruflichen Motiven nennt fast die Hälfte $(48,7 \%$ ) (also rund jeder vierte Befragte insgesamt) das konkrete Motiv, mit dem Umzug in die Nähe des vorhandenen Arbeitsplatzes zu ziehen (ohne Tabelle). Weitere $29,7 \%$ nennen den Antritt eines neuen Arbeitsplatzes, $11,9 \%$ den Antritt eines Studiums oder einer Ausbildung. Von jeweils 4-6\% der Betreffenden werden Eintritt in die Rente, Aufnahme einer selbstständigen Tätigkeit oder sonstige berufliche Gründe genannt. Kleinräumliche Ausbildungswanderungen (etwa bei den Eltern ausziehende Studierende) sind also in den beruflich motivierten Wanderungen nicht dominant.

Tab. 1 Wanderungsmotive

\begin{tabular}{|c|c|c|c|c|c|c|c|}
\hline & \multirow[t]{2}{*}{$\begin{array}{l}\text { Familiäre/Persönliche } \\
\text { Gründe }\end{array}$} & \multirow[t]{2}{*}{$\begin{array}{l}\text { Bildung von } \\
\text { Wohneigentum }\end{array}$} & \multirow[t]{2}{*}{$\begin{array}{l}\text { Vorherige } \\
\text { Wohnung/Haus }\end{array}$} & \multirow[t]{2}{*}{$\begin{array}{l}\text { Vorheriges } \\
\text { Wohngebiet }\end{array}$} & \multirow[t]{2}{*}{$\begin{array}{l}\text { Berufliche } \\
\text { Gründe }\end{array}$} & \multicolumn{2}{|c|}{$\begin{array}{l}\text { Darunter: Zielort des } \\
\text { Umzugs... }\end{array}$} \\
\hline & & & & & & $\begin{array}{l}\text { Ober- } \\
\text { zentrum }\end{array}$ & $\begin{array}{l}\text { Anderer } \\
\text { Ort }\end{array}$ \\
\hline Ausschlaggebend & 52,4 & 10,7 & 23,0 & 17,8 & 23,6 & 35,4 & 18,1 \\
\hline Hat auch eine Rolle gespielt & 26,6 & 6,0 & 27,9 & 31,1 & 23,1 & 22,7 & 23,4 \\
\hline Nicht wichtig & 20,9 & 83,3 & 49,1 & 51,1 & 53,3 & 41,9 & 58,5 \\
\hline Gesamtsumme & 100,0 & 100,0 & 100,0 & 100,0 & 100,0 & 100,0 & 100,0 \\
\hline$n$ & 837 & 832 & 792 & 785 & 783 & 229 & 508 \\
\hline
\end{tabular}

Unterschied zwischen Zielorten des Umzugs signifikant (Chi-Quadrat-Test, $p=0,05)$. Cramér’s V=0,196 


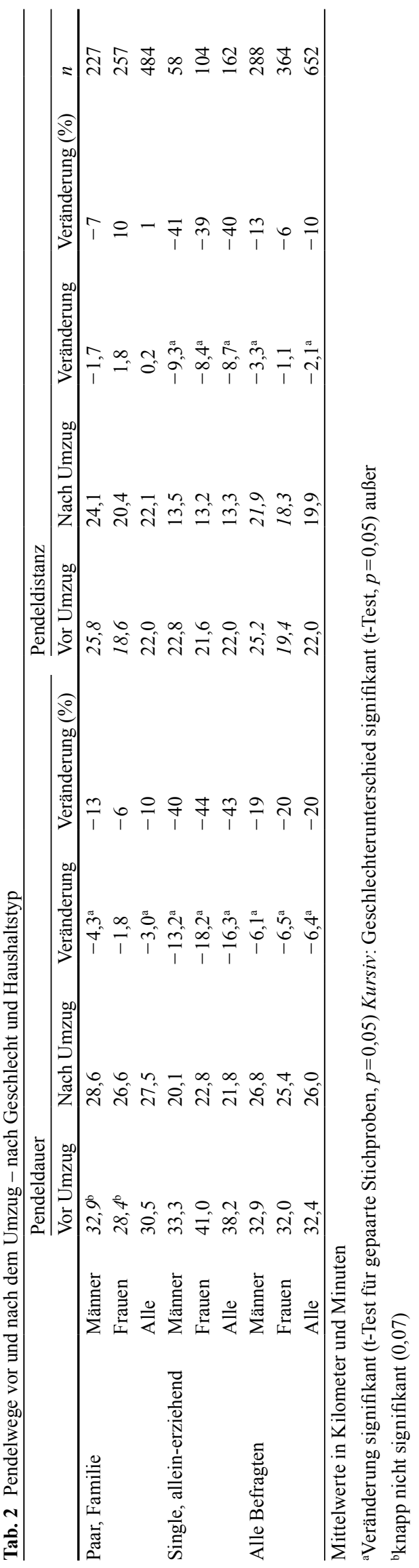

Bei Umzügen in eins der nahegelegenen Oberzentren Dortmund, Bochum, Essen oder Wuppertal sind berufliche Motive noch häufiger (35,4\% ausschlaggebend, 22,7\% hat auch eine Rolle gespielt, Tabelle 1 rechts). Daneben treten berufliche Motive vor allem bei Umzugsdistanzen ab ca. $20 \mathrm{~km}$ auf (37,6\% ausschlaggebend, 20,4\% hat auch eine Rolle gespielt). Aber auch bei Umzügen über kürzere Distanzen spielen sie für $40 \%$ der Befragten zumindest eine Nebenrolle.

Bei 21,3\% der Befragten befindet sich nach dem Umzug der Arbeitsplatz in einem anderen PLZ-Gebiet als vorher. Dies ist die Untergrenze der Häufigkeit von Arbeitsplatzwechseln, die mit dem Umzug in einem mittelbaren oder direkten Bezug stehen, weil Arbeitsplatzwechsel innerhalb eines PLZ-Gebiets nicht erfasst werden. Nach Umzügen in ein Oberzentrum ändert sich die Lage des Arbeitsplatzes erwartungsgemäß etwas - aber nicht wesentlich - häufiger $(24,6 \%)$.

Bei Frauen geht der Umzug etwas häufiger $(24,6 \%)$ mit einer räumlichen Verlagerung des Arbeits- oder Ausbildungsplatzes einher als bei Männern (18,1\%). Dies bedeutet jedoch nicht, dass die Umzüge den Arbeitsplatzwechseln der Frauen folgen. In diesem Fall müssten vor allem deren Pendelwege mit dem Umzug kürzer werden; tatsächlich werden jedoch vor allem die Pendelwege der Männer kürzer (siehe unten).

\subsection{Pendeln vor und nach dem Umzug}

Frauen legen sowohl vor als auch nach dem Umzug kürzere Pendelwege zurück als Männer (Tabelle 2). Dies gilt für die Distanz wie auch für die Pendeldauer, für letztere allerdings nur minimal und nicht signifikant, was die bekannte Beobachtung der Verkehrsforschung (Crane 2007) bestätigt, dass Frauen sich langsamer fortbewegen (Differenz ca. $6 \mathrm{~km} / \mathrm{h}$, t-Test signifikant, $p=0,05$ ). Dies ist auf ihre stärkere Nutzung des ÖPNV und das häufigere Gehen zu Fuß zurückzuführen.

Die kürzeren Distanzen der Frauen lassen zwei mögliche Schlussfolgerungen zu. Die gängige Interpretation der feministischen Verkehrsforschung wäre, dass die Frau in der Wahl des Arbeitsortes stärker an den Wohnort gebunden ist als der Mann. Die umgekehrte, ,klassische' Interpretation interpretiert einen langen Pendelweg als (Zeit-) Verlust. Demzufolge würden Paare miteinander um einen kurzen individuellen Pendelweg, konkurrieren', und ein kurzer Weg der Frau würde bedeuten, dass diese sowohl vor dem Umzug (in Hagen) als auch nach der Wanderung die Wohnstandortentscheidung dominiert.

Zur weiteren Interpretation helfen eine Unterscheidung nach Haushaltstypen und der Blick auf Personen in Partnerschaften. Bei Singles werden sowohl Pendeldistanzen als auch Zeitaufwand durch den Umzug etwa gleich stark 
reduziert (Distanz um 40\%, Zeit um 43\%). Dies gilt im Wesentlichen für Frauen und Männer gleichermaßen. Bei Paarhaushalten (einschließlich Familien) stellt sich die Sache anders dar. Diese reduzieren - individuell und auf der Haushaltsebene - ihren Pendelaufwand deutlich weniger als Singles. Dabei ,gewinnen' die Männer. Bei diesen wird der Zeitaufwand durch den Umzug stärker (und signifikant) reduziert als bei den Frauen. Bei Männern reduziert sich auch die Pendeldistanz, bei Frauen nimmt sie dagegen zu. Dadurch gleicht sich der deutliche Geschlechterunterschied in den Pendeldistanzen vor dem Umzug durch den Umzug etwas an. Dies gilt ähnlich auch für die Pendeldauer. Diese relative Angleichung wäre nach der Lesart der feministischen Verkehrsforschung als (relative) Emanzipation durch den Umzug zu interpretieren. Die deutliche Reduktion der Pendeldauer $(-13 \%)$ und Pendeldistanz $(-7 \%)$ der Männer bedeutet jedoch, dass Paare eher zum Arbeitsplatz des Mannes ziehen. Dies unterstützt die „Trailing-wife“-These. Da auch der Pendelzeitaufwand der Paare insgesamt (also auf der Paarebene) reduziert wird, folgt die Standortverlagerung typischerweise durchaus einer (neoklassischen) Haushaltslogik der Optimierung des Wohnstandortes für den Haushalt insgesamt. Dies geschieht aber - entgegen der in der neoklassischen Theorie unterstellten Geschlechterneutralität - zum stärkeren Vorteil des Mannes.

\subsection{Die Rolle des Arbeitsplatzwechsels bei Frauen}

Den theoretischen Überlegungen zufolge sollten Frauen nach dem Umzug einen kurzen Arbeitsweg aufweisen, sofern sie mit bzw. nach dem Umzug ihren Arbeitsplatz wechseln, zum einen aufgrund des ,spatial entrapment“ durch Haushaltspflichten und geringere Mobilität, zum anderen aufgrund ihrer in der Regel geringeren Spezialisierung und der damit einhergehenden geringeren Segregation der Arbeitsplätze. Wenn sie den Arbeitsplatz nicht wechseln, sollte ihr Arbeitsweg eher länger werden. Betrachtet werden hier jeweils Frauen und - zum Vergleich - Männer, die als Paare bzw. Familien leben.

Unabhängig vom Geschlecht wird der Pendelzeitaufwand des Haushalts bei Paaren bzw. Familien etwa gleichermaßen reduziert, ob einer der Arbeitsplätze gewechselt wird $(-14 \%)$ oder nicht $(-11 \%)$. Die Pendeldistanz nimmt bei einem Arbeitsplatzwechsel sogar geringfügig zu. Dies spricht zunächst für eine Optimierung des Zeitaufwandes durch den Umzug, unabhängig davon, ob mit dem Umzug ein Arbeitsplatzwechsel einhergeht. Männer und Frauen reduzieren dabei ihren Zeitaufwand etwa im gleichen Ausmaß ( -12 vs. $-11 \%)$, wenn sie ihren Arbeitsplatz nach dem Umzug wechseln. Die Distanz nimmt dann bei Männern etwas ab, bei Frauen etwas zu. 
Bei einem Wechsel des Arbeitsplatzes stellt sich der Geschlechtervergleich allerdings ganz anders dar (Tabelle 3). Wechselt der Mann den Arbeitsplatz, wird seine Pendeldauer durch den Umzug stark reduziert $(-33 \%)$. Wechselt eine Frau den Arbeitsplatz, bleibt ihre Pendeldauer dagegen gleich. Gleichzeitig geht beim Arbeitsplatzwechsel des Mannes auch dessen Distanz deutlich zurück $(-13 \%)$, beim Arbeitsplatzwechsel der Frau nimmt deren Distanz jedoch deutlich zu (+17\%). Dies sind deutliche Hinweise darauf, dass die Haushalte eher zum neuen Arbeitsplatz des Mannes ziehen, wobei die Frau - selbst wenn sie den Arbeitsplatz wechselt - zeitlich nicht profitiert und im Hinblick auf die zurückzulegende Distanz sogar für den kürzeren Weg des Mannes ,bezahlt' (Veränderung der Distanzen aufgrund der kleinen Teilstichproben - 36 Männer, 52 Frauen - nicht signifikant).

Nun ist nicht gesagt, dass bei diesen Haushalten jeweils beide Partner ihren Arbeitsplatz wechseln. Eine entsprechende Subgruppenanalyse ist aufgrund der begrenzten Stichprobe nicht möglich. Dennoch zeigen die Ergebnisse, dass die Umzüge nicht den Arbeitsplätzen der Frauen folgen, wohl aber den Arbeitsplätzen der Männer. Dies stützt deutlich die These der „trailing wives“ und einer Optimierung des Wohnstandortes zum Vorteil der Karriere (Arbeitsplatzwechsel!) des Mannes. Gleichzeitig stellen die Ergebnisse aber die feministische Interpretation kurzer Pendelwege als Indikator für eine Benachteiligung von Frauen in Frage. Die Ergebnisse sprechen deutlich dagegen, dass Frauen sich in ihrem Suchradius einschränken, wenn sie nach dem Umzug einen neuen Arbeitsplatz annehmen. Im Gegenteil: Selbst wenn eine Frau einen neuen Arbeitsplatz annimmt, pendelt sie tendenziell eher weiter als vorher.

\subsection{Die Konfiguration von Wohnort und Arbeitsorten bei Paarhaushalten}

Wie verändert sich die Konfiguration von Wohnort und Arbeitsorten erwerbstätiger Paare nach dem Umzug? Tabelle 4 zeigt die Mittelwerte und Mediane des Winkels, den der Wohnort zwischen den Arbeitsorten aufspannt, vor und nach dem Umzug. Dieser Winkel wird nach dem Umzug größer; demnach tendieren die Haushalte dazu, mit dem Umzug räumlich zwischen die beiden Arbeitsplätze zu ziehen. Die Pendeldistanzen bei Paaren werden jedoch nach dem Umzug nicht kürzer (siehe Kapitel 4.2). Demnach wird die Wohnstandortwahl - der Umzug zwischen die beiden Arbeitsplätze - als Strategie benutzt, damit die Distanzen zumindest nicht länger werden, etwa wenn einer der Arbeitsorte gewechselt wird.

Diese Analyse lässt sich mit den vorhandenen Daten allerdings nur für Paare vornehmen, bei denen der Wohnort und beide Arbeitsorte in drei verschiedenen PLZ-Gebieten liegen. Tabelle 5 zeigt, dass der Anteil der Haushalte
Tab. 4 Winkel zwischen Wohnort und Arbeitsorten (Paarhaushalte mit zwei Erwerbstätigen)

\begin{tabular}{llll}
\hline & \multicolumn{3}{l}{ Winkel Wohnen-Arbeitsplätze (Grad) } \\
\cline { 2 - 4 } & Vor Umzug & Nach Umzug & Veränderung \\
\hline Mittelwert & $73,6^{\circ}$ & $87,7^{\circ}$ & $14,2^{\circ}$ \\
Median & $59,4^{\circ}$ & $79,1^{\circ}$ & $11,1^{\circ}$ \\
$n$ & 116 & 116 & 116 \\
\hline
\end{tabular}

Veränderung des Mittelwerts (knapp) nicht signifikant (t-Test für gepaarte Stichproben, $p=0,06$ )

Veränderung des Medians signifikant (Wilcoxon-Test für gepaarte Stichproben, $p=0,05$ )

Der Wert für „Median der Veränderung“ entspricht nicht der Differenz der anderen Mediane

Tab. 5 Kombination der Lage von Wohnort und Arbeitsorten (Paarhaushalte mit zwei Erwerbstätigen)

\begin{tabular}{lcc}
\hline Lage Wohnort und Arbeitsorte & Vor Umzug & Nach Umzug \\
\hline $\begin{array}{l}\text {.. in unterschiedlichen } \\
\text { PLZ-Gebieten }\end{array}$ & 78,2 & 73,7 \\
$\ldots$ im gleichen PLZ-Gebiet & 15,0 & 20,8 \\
(Wohnort, ein Arbeitsort) & & \\
$\ldots$. im gleichen PLZ-Gebiet & 6,8 & 5,5 \\
(Wohnort, beide Arbeitsorte) & & \\
Summe & 100,0 & 100,0 \\
$n$ & 353 & 361 \\
\hline
\end{tabular}

Veränderung signifikant (Chi-Quadrat-Anpassungstest, $p=0,01$ )

zunimmt, bei denen der Wohnort und ein Arbeitsort im gleichen PLZ-Gebiet liegen. Auch dies spricht für eine strategische, am Arbeitsplatz eines Partners ausgerichtete Wohnstandortwahl der Paarhaushalte. Dabei lässt sich in der Tendenz auf niedrigem Niveau eine Zunahme der Männer beobachten, die nach dem Umzug im gleichen PLZ-Gebiet arbeiten, in dem sie auch wohnen (ohne Tabelle). Dies bestätigt die leicht stärkere Orientierung der Wohnstandortwahl am Arbeitsplatz der Männer.

\subsection{Die individuelle Pendeldistanz}

In den folgenden Regressionsmodellen werden Einflussfaktoren der Pendeldistanz nach der Abwanderung aus der Kernstadt untersucht. Der Fokus liegt auf Faktoren, die sich in der Literatur bzw. der theoretischen Herleitung als bedeutsam erwiesen haben (Geschlecht, Haushaltsstruktur, Sozioökonomie, räumliches Umfeld, Umzugsmotiv und -distanz, Pfadabhängigkeit). Dabei werden stufenweise mehr Variablen aufgenommen, um zu beobachten, wie sich die auftretenden Effekte verändern (Tabelle 6).

Ein erstes Modell (Modell 1), das ausschließlich Geschlechterunterschiede berücksichtigt, zeigt die signifikant kürzeren Pendelwege der Frauen. Bei Kontrolle des Einkommens und der Bildung (Modell 2) wird der Geschlechterunterschied insignifikant. Demzufolge ist die kürzere Distanz der Frauen in starkem Maße auf Statusbzw. ökonomische Unterschiede zurückzuführen. Die 


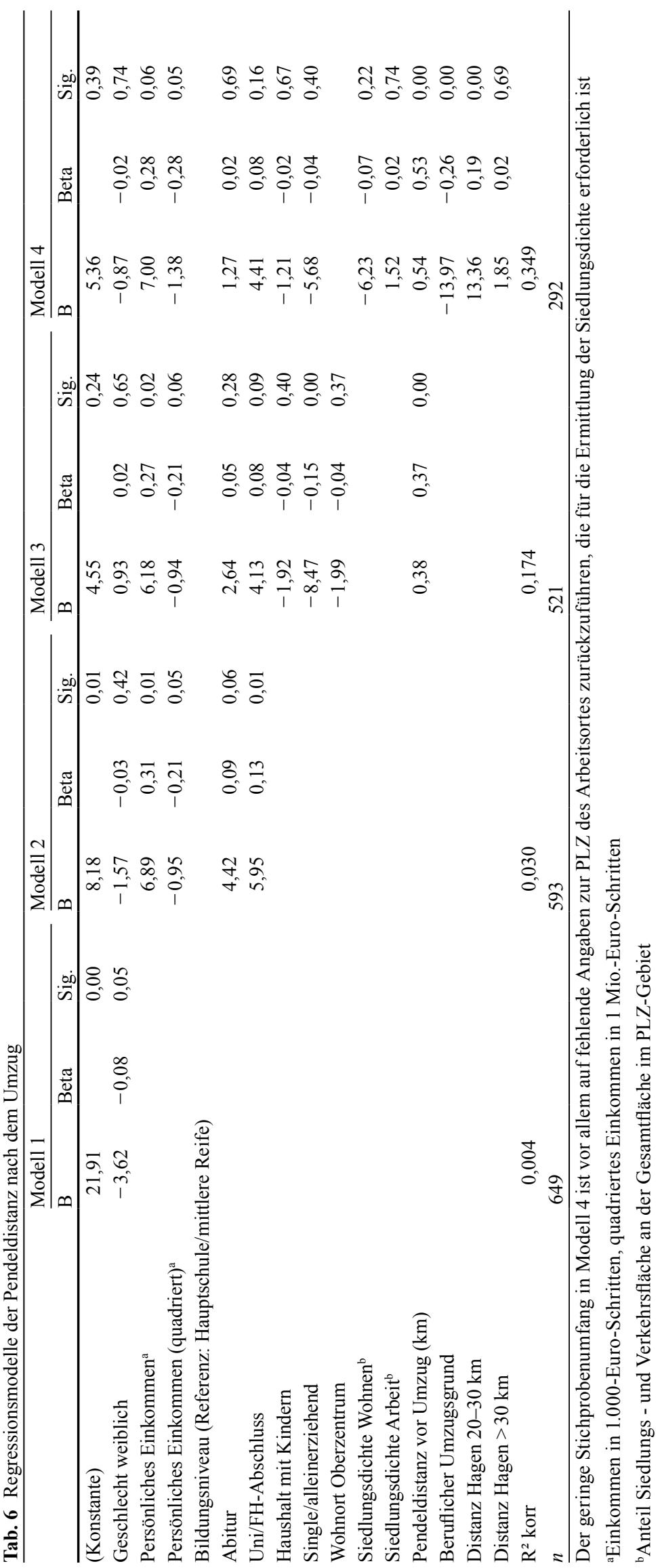


Beziehung der Pendeldistanz zum Einkommen ist dabei nicht-linear, wie sich an der quadratischen Funktion zeigt. Modell 3 zeigt darüber hinaus signifikant kürzere Pendelwege von Personen ohne Partner im Haushalt (Singles, Alleinerziehende). Das Modell verdeutlicht auch die starke Pfadabhängigkeit des Pendelns: Lange Pendelwege bereits vor dem Umzug sind stark mit langen Pendelwegen auch nach dem Umzug assoziiert. Räumliche Unterschiede sind nicht signifikant, deuten jedoch plausiblerweise auf kürzere Pendeldistanzen, wenn der Umzug in ein Oberzentrum erfolgte. Modell 4 zeigt zusätzlich die deutlich längeren Pendeldistanzen in einer Entfernung von 20-30 km um Hagen, nicht aber bei größeren Entfernungen (die typischerweise mit Orientierungen an anderen Kernen einhergehen). Darüber hinaus zeigt das Modell die deutlich kürzeren Pendeldistanzen, wenn der Umzug beruflich motiviert war. Neben den drei wichtigen Einflussgrößen Umzugsmotiv, Entfernung nach Hagen und Pendeldistanz vor dem Umzug werden nahezu alle anderen Einflussgrößen insignifikant.

\subsection{Die Haushalts-Pendeldistanz}

In Tabelle 7 wird die Summe der Pendeldistanzen zweier Partner untersucht. Diese lässt - neben der räumlichen Konfiguration von Wohnort und Arbeitsorten - weitere Schlussfolgerungen für die Pendeleffizienz der Wohnstandortwahl auf der Haushaltsebene zu.

Das quadrierte Einkommen zeigt auf der Haushaltsebene keine nennenswerten Effekte. Es wird deshalb aus der weiteren Modellierung ausgeschlossen. Demnach scheint hier ein eher linearer, positiver Zusammenhang zur Pendeldistanz vorzuliegen. Dies ist zum Teil darauf zurückzuführen, dass in Haushalten mit höherem Einkommen häufig zwei Erwerbstätige leben (mit entsprechend hoher Haushalts-Pendeldistanz). Dieser Zusammenhang erklärt auch einen Teil der stark reduzierten Distanzen bei Singles bzw. Alleinerziehenden. Diese neigen auch stärker als Paarhaushalte dazu, mit dem Umzug ihre individuelle Distanz zum Arbeitsplatz zu reduzieren (siehe Tabelle 2).

Arbeitsplätze in urbanen Lagen (hohe Siedlungsdichte am Arbeitsort) sind mit längeren Pendelwegen verbunden. Darin drückt sich ein wenig beachteter Aspekt von Urbanität aus, nämlich die großen Pendlereinzugsbereiche von Städten mit der Folge langer Wege für einpendelnde Erwerbstätige. Dieser Zusammenhang verliert die Signifikanz in den folgenden Modellen, bleibt jedoch stark positiv.

Die Pfadabhängigkeit des Pendelns (HaushaltsPendeldistanz vor dem Umzug) erweist sich wie auf der individuellen Ebene als starker Einfluss (Modell 6). In Modell 7 wird auch die Veränderung des Winkels nach dem Umzug berücksichtigt, der durch Wohnort und Arbeitsorte aufgespannt wird. Diese Veränderung steht in einer signifikant positiven Beziehung zur Haushalts-Pendeldistanz.
Haushalte mit besonders weiten Pendeldistanzen neigen demnach besonders stark dazu, ihren Wohnstandort so zu verändern, dass sich die langen Wege in der Summe beider Partner verringern, indem sie zwischen die beiden Arbeitsplätze ziehen. Dieser Zusammenhang ist recht stark; der standardisierte Effekt (Beta) liegt etwa auf dem Niveau des Einkommenseffektes. In Modell 8 sind - ähnlich wie im Modell 4 der individuellen Pendeldistanz - nur noch die drei bedeutenden Einflussgrößen Umzugsmotiv, Entfernung nach Hagen und Pendeldistanz vor dem Umzug signifikant. Dies liegt auch an der kleinen Teilstichprobe.

\subsection{Die Pendeldifferenz in Partnerschaften}

Abschließend wird untersucht, ob sich aus Geschlechterverhältnissen die Differenz der Pendelwegelänge zwischen zwei Partnern P1 und P2 erklären lässt. So ist nach dem Stand der Literatur zu erwarten, dass diese Differenz d(P1) - d(P2) aufgrund der kürzeren Pendelwege von Frauen negativ ist, wenn die erste Person (P1) die Frau ist. $\mathrm{Zu}$ erwarten ist auch, dass die Differenz noch stärker negativ ist, wenn das Einkommen der Frau besonders gering und/ oder das Einkommen des Mannes besonders hoch ist oder wenn es sich um einen Haushalt mit Kindern handelt.

Der in Modell 9 (Tabelle 8) auftretende starke Geschlechtereffekt bestätigt einmal mehr die kürzeren Pendelwege von Frauen, hier allerdings relativ zu ihren Partnern. Eine lange Distanz (relativ zum Partner) ist vor allem bei hoher Qualifikation der befragten Person zu erwarten. Entsprechend beeinflusst ein akademischer Abschluss die Differenz der Pendeldistanzen in Richtung des Befragten; wenn der Partner oder die Partnerin einen akademischen Abschluss besitzt, ist es umgekehrt. Umgekehrt allerdings reduziert eine leitende berufliche Position tendenziell die Differenz der Pendeldistanzen (nicht signifikant). Hier geht also der Statuseffekt in die gegenteilige Richtung einer relativ kurzen Distanz bei Personen in Führungspositionen, ${ }^{4}$ möglicherweise aufgrund von deren Dominanz in der Wohnstandortentscheidung.

Starke Einflüsse gehen von der Lage der Arbeitsorte aus. Diese fügen eine weitere interessante Facette zu den bereits gefundenen räumlichen Effekten hinzu. Während oben (Modell 5) festgestellt wurde, dass eine urbane Lage von Arbeitsplätzen mit längeren Wegen einhergeht, reduziert eine solche Lage die Differenz zwischen den Pendel-

\footnotetext{
${ }^{4} \mathrm{Da}$ es bei der Differenz zwischen zwei Partnern um ein ,relatives Verhalten“ geht, wurden bezüglich des sozialen Status auch relative Variablen getestet (anteiliger Beitrag der Partner zum Haushaltseinkommen, Einkommensdifferenz zwischen Partnern, Relation der beruflichen Position der Partner zueinander, relatives Bildungsniveau). In der statistischen Varianzaufklärung erwiesen sich diese jeweils gegenüber den letztlich verwendeten separaten Varianten für die beiden Partner als unterlegen.
} 


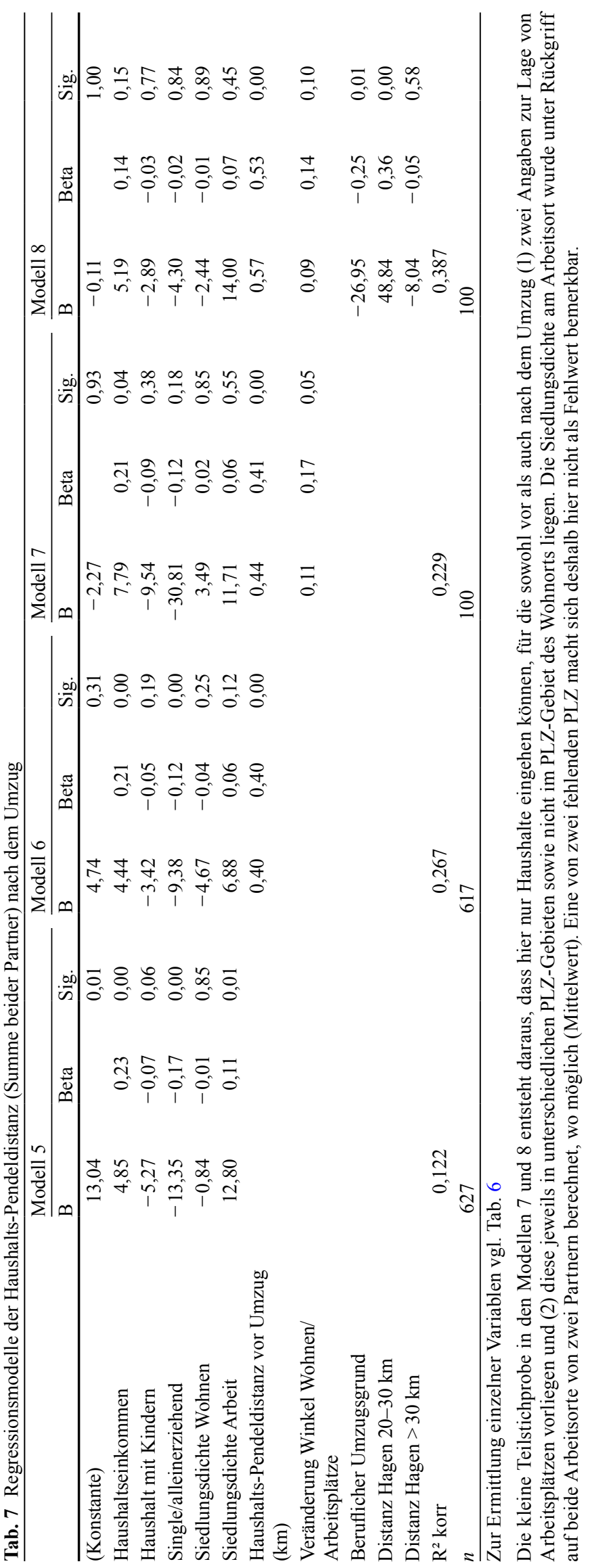


Tab. 8 Regressionsmodelle der Differenz der Pendeldistanz zwischen zwei Partnern nach dem Umzug

\begin{tabular}{|c|c|c|c|c|c|c|}
\hline & \multicolumn{3}{|l|}{ Modell 9} & \multicolumn{3}{|c|}{ Modell 10} \\
\hline & $\overline{\mathrm{B}}$ & Beta & Sig. & $\overline{\mathrm{B}}$ & Beta & Sig. \\
\hline$\overline{\text { (Konstante) }}$ & 5,99 & & 0,51 & $-5,67$ & & 0,51 \\
\hline Geschlecht weiblich & $-13,35$ & $-0,18$ & 0,00 & $-7,12$ & $-0,09$ & 0,10 \\
\hline Persönliches Einkommen & 1,84 & 0,05 & 0,76 & 9,64 & 0,27 & 0,08 \\
\hline Persönliches Einkommen (quadriert) & $-0,25$ & $-0,04$ & 0,83 & $-1,77$ & $-0,26$ & 0,08 \\
\hline Befragter: Uni-/FH-Abschluss & 11,09 & 0,15 & 0,03 & 8,20 & 0,10 & 0,08 \\
\hline Partner: Uni-/FH-Abschluss & $-12,09$ & $-0,16$ & 0,02 & $-11,41$ & $-0,14$ & 0,02 \\
\hline Befragter: Leitende Position im Beruf & $-8,89$ & $-0,10$ & 0,10 & $-10,02$ & $-0,11$ & 0,05 \\
\hline Partner: Leitende Position im Beruf & 7,20 & 0,09 & 0,16 & 8,02 & 0,09 & 0,10 \\
\hline Siedlungsdichte Wohnen & $-3,07$ & $-0,02$ & 0,72 & $-1,70$ & $-0,01$ & 0,83 \\
\hline Befragter: Siedlungsdichte Arbeit & $-20,57$ & $-0,17$ & 0,01 & $-3,19$ & $-0,02$ & 0,66 \\
\hline Partner: Siedlungsdichte Arbeit & 21,11 & 0,17 & 0,01 & 4,62 & 0,04 & 0,53 \\
\hline Differenz Pendeldistanz Befragter-Partner vor Umzug & & & & 0,61 & 0,59 & 0,00 \\
\hline $\mathrm{R}^{2}$ korr & 0,073 & & & 0,412 & & \\
\hline$n$ & 283 & & & 233 & & \\
\hline
\end{tabular}

Zur Ermittlung einzelner Variablen vgl. Tab. 6

distanzen zweier Partner. Demnach ist eine urbane Lage des Arbeitsplatzes des einen Partners mit vergleichsweise (noch) längeren Wegen des anderen Partners verbunden. Die Lage von dessen Arbeitsort wirkt sich spiegelbildlich aus. Die Siedlungsdichte am Wohnort hat dagegen keinen nennenswerten Einfluss.

Sowohl die beobachteten Statuseinflüsse als auch die räumlichen Effekte gelten für beide Partner in einem Haushalt gleichermaßen, sind also praktisch geschlechtsneutral. Diese Interpretation lässt sich auch dadurch stützen, dass keine nennenswerten Interaktionen zwischen Geschlecht und Kindern oder zwischen Geschlecht und Einkommen gefunden wurden. Demnach wirken sich Ressourcen und Zwänge im hier untersuchten Kontext kaum geschlechtsspezifisch aus.

Modell 10 zeigt gegenüber Modell 9 einige wichtige Variationen. Die Pfadabhängigkeit der untersuchten Variablen (Einfluss der Differenz der Pendeldistanz vor dem Umzug) erweist sich einmal mehr als sehr stark. Pfadabhängigkeit des Verhaltens wird häufig im Sinne von Routine oder ,Ermüdung ' des Verhaltens interpretiert. Diese Interpretation greift hier zu kurz, denn hier impliziert die gefundene Pfadabhängigkeit, dass die Verteilung der Pendeldistanz bei Paaren (Wer hat den längeren Weg?) auch nach einem Umzug - also einer Umbruchsituation - stabil bleibt.

Bezüglich der Bildung und der beruflichen Position bleiben die Befunde weitgehend stabil. Die Einflüsse der Siedlungsdichte verlieren stark an Gewicht (auch wenn sie in der Richtung gleich bleiben). Das Einkommen gewinnt deutlich an Einfluss. Wenngleich die Einkommenseffekte nicht ganz das Signifikanzniveau erreichen, zeigt sich doch plausibel eine umgekehrte U-Form. Das heißt, mit zunehmendem Einkommen einer Person wird deren Pendeldistanz relativ zum Partner länger; etwa $a b$ einem
Einkommen von 2.700 Euro netto dreht sich das Verhältnis allerdings wieder um, vermutlich weil sehr gut verdienende Partner die Wohnstandortwahl stärker dominieren, ähnlich wie es auch im Effekt einer leitenden beruflichen Position zum Ausdruck kommt.

\section{Resümee}

Dieser Beitrag untersuchte die Pendeldistanzen und ergänzend die Dauer der Pendelwege im Kontext von Randwanderung und Geschlechterverhältnissen im Umland der Stadt Hagen, d. h. in einer polyzentralen, altindustriellen Region. Die Ergebnisse zeigen, dass auch innerregionale Wanderungen häufig mit beruflichen Motiven verbunden sind. Obwohl hier Abwanderungen aus der Stadt in das Umland untersucht wurden, zeigt sich eine Reduktion des Pendelzeitaufwands durch den Umzug, vermutlich weil es sich aufgrund der Polyzentralität nur teilweise um klassische Randwanderungen handelt.

Die geschlechtsspezifischen Veränderungen der Pendelwegelängen nach dem Umzug sprechen für die Orientierung des Wohnstandorts am Arbeitsplatz des Mannes, wobei die Frauen tendenziell die „Trailing-wife“-Position einnehmen. Gleichzeitig scheinen die Dinge komplizierter zu liegen als in den Hypothesen der feministisch geprägten Verkehrsforschung angenommen. Denn gerade bei Paaren zeigt sich ein räumlich überdurchschnittlich eingegrenztes Pendeln (kurze Wege) der Frauen nach dem Umzug nicht. Dies ist vermutlich durch die polyzentrale Struktur des Untersuchungsraumes bedingt, der bei der gegebenen hohen Motorisierung vielfältige Optionen des Arbeitsmarktes bietet.

Auf der Haushaltsebene ist erkennbar, dass die Wanderungen insgesamt mit dem Versuch einer Ver- 
kürzung der Pendelwege einhergehen. Dies gilt für Singles - die keine Kompromisse eingehen müssen - mehr als für Paarhaushalte. Das drückt sich nicht nur in der veränderten Länge der Pendelwege aus (mehr in Bezug auf die Dauer als auf die Distanz), sondern auch in der räumlichen Konfiguration von Wohnen und Arbeiten bei erwerbstätigen Paaren. Diese Befunde zeigen, dass Paare bemüht sind, entweder näher zu einem der beiden Arbeitsplätze oder zwischen die beiden Arbeitsplätze zu ziehen.

In einem Ring von $20-30 \mathrm{~km}$ um Hagen sind die Wohnstandortentscheidungen allerdings im Vergleich $\mathrm{zu}$ Wanderungen in das nähere Umland $(<20 \mathrm{~km})$ mit deutlich längeren Pendeldistanzen nach dem Umzug verbunden, was den klassischen Befunden der Suburbanisierungsforschung entspricht. Noch größere Wanderungsdistanzen resultieren nicht in längeren Pendelwegen, weil diese Wanderungen gerade in der polyzentrischen Struktur der Region häufiger mit Arbeitsplatzverlagerungen verbunden sind.

In den Pendeldistanzen zeigen sich erhebliche Pfadabhängigkeiten. Offenbar gibt es stabile Toleranzniveaus gegenüber Distanzen sowohl auf der Ebene individueller Personen als auch von Paaren, die auch einen Umzug überdauern. Ebenso ist die Geschlechterverteilung der Distanzen bei Paaren über einen Umzug hinweg stabil.

Nicht alle Ergebnisse sind im Hinblick auf ihre statistische Belastbarkeit befriedigend. Dennoch zeigt der Beitrag auch in methodischer Hinsicht Wege zur Weiterentwicklung der Pendler- und weiteren Verkehrsforschung auf. Zum einen ist hier die Verwendung des Winkels zwischen Wohnund Arbeitsorten im Anschluss an Surprenant-Legault/ Patterson/El-Geneidy (2013) zu nennen, der die räumliche Konfiguration dieser Orte abbildet. Zweitens ließ sich, wenn auch sehr vereinfacht, mithilfe der Postleitzahl die Siedlungsstruktur am Wohnort und den Arbeitsorten kleinräumlich abbilden. Gerade bezüglich des Arbeitsortes liegen hierzu äußerst wenige Erkenntnisse aus Deutschland vor. Drittens wurde - eher ungewöhnlich - die Haushalts-Pendeldistanz als weiteres $\mathrm{Ma} ß$ der Pendeleffizienz untersucht. Viertens lässt die Kontrolle des persönlichen Einkommens Aussagen über die Verteilung ökonomischer Ressourcen bei Paaren zu, was mit nationalen Mobilitätsdaten in der Regel nicht möglich ist.

Für die künftige Forschung ergeben sich einige Schlussfolgerungen. Erstens würden geokodierte Daten der Wohn- und Arbeitsorte erhebliche Möglichkeiten für die Mobilitätsforschung eröffnen. Die Geokodierung sollte dabei deutlich genauer sein, als es hier möglich war. Zweitens sollte die Pendlerforschung neben den üblichen Indikatoren wie Pendeldistanz und -dauer auf der Individualebene auch andere Maße der Pendeleffizienz betrachten (Haushalts-Pendeldistanz, Konfiguration von Wohn- und Arbeitsorten). Drittens lassen sich für die Untersuchung von Geschlechterverhältnissen in Wohnstandortwahl und Pendelverhalten (bzw. allgemeiner: Mobilität) Variablen gewinnbringend einsetzen, die auf Interaktionen innerhalb von Haushalten beruhen, etwa Personenmerkmale des Partners und anderer Haushaltsmitglieder oder persönliche ökonomische Ressourcen.

Inhaltlich bestätigen die Ergebnisse die Hypothese der „trailing wives“, stellen aber die Interpretation kurzer Pendelwege als Benachteiligung der Frauen in Frage. Daraus ergeben sich Notwendigkeiten zur Weiterentwicklung der theoretischen Annahmen einer genderorientierten Mobilitätsforschung. Eine systematische Erforschung des Einflusses der regionalen Zentrenstruktur (mono- vs. polyzentrisch) wäre dabei ebenfalls eine wichtige Aufgabe.

Insgesamt sprechen die Ergebnisse für sehr komplexe Beziehungen zwischen Geschlecht, sozialem Status, Wohnstandortwahl und Pendelverhalten. Aus planungspolitischer Sicht tragen sie zunächst zu einem besseren Verständnis dieser Beziehungen und zur Entwicklung geschlechtersensibler Strategien bei. Konkret machen sie deutlich, dass Haushalte durchaus bemüht sind, ihren Pendelaufwand auch nach Randwanderungen zu begrenzen. Die darin zum Ausdruck kommende Rationalität kann gerade in Zeiten steigender Verkehrskosten zur Ansprache wanderungswilliger Haushalte genutzt werden.

Die Ergebnisse verdeutlichen auch, dass in einer polyzentralen Region wie der hier untersuchten vielfältige Optionen für Haushalte bestehen, die Abwanderung aus der Stadt mit einer alltagsverträglichen (Re)-Organisation der Erwerbstätigkeit zu verbinden. In Paarhaushalten geht dies allerdings häufig auf Kosten erwerbstätiger Frauen - nicht im Sinne einer „räumlichen Falle“, sondern im Sinne einer Reduzierung des Pendelaufwands vor allem (oder nur) für den Mann.

Danksagung Dieser Beitrag entstand im Rahmen des von der Deutschen Forschungsgemeinschaft geförderten Projekts „Alltag im Wandel des Geschlechterverhältnisses: Aktivitäten, Wege, Verkehrsmittel und Zeitverwendung“"(2009-2016). 


\section{Anhang}

Tab. 9 Deskriptive Statistiken der metrischen Variablen in den Regressionsanalysen

\begin{tabular}{lcc}
\hline & Mittelwert & Standardabweichung \\
\hline Pendeldistanz nach dem Umzug (individuell) $(\mathrm{km})$ & 19,9 & 23,8 \\
Haushalts-Pendeldistanz nach dem Umzug $(\mathrm{km})$ & 31,6 & 32,4 \\
Differenz der Pendeldistanz Befragter-Partner nach dem Umzug (km) & 0,12 & 38,5 \\
Pendeldistanz vor dem Umzug (individuell) (km) & 22,3 & 23,0 \\
Haushalts-Pendeldistanz vor dem Umzug (km) & 58,4 \\
Differenz der Pendeldistanz Befragter-Partner vor dem Umzug (km) & 0,87 \\
Persönliches Einkommen (netto, in 1.000 €) & 1,87 \\
Persönliches Einkommen (netto, quadriert/1 Mio. €) & 4,59 & 37,1 \\
Siedlungsdichte am Wohnort nach dem Umzug & 0,37 & 5,05 \\
Siedlungsdichte am Arbeitsort nach dem Umzug & 0,53 \\
Siedlungsdichte am Arbeitsort des Partners nach dem Umzug (jeweils: Anteil Siedlungs- und Ver- & 0,29 \\
kehrsfläche im PLZ-Gebiet) & 0,52 \\
Veränderung des Winkels Wohnen/Arbeitsplätze nach dem Umzug (Grad) & 0,30
\end{tabular}

Tab. 10 Deskriptive Statistiken der kategorialen Variablen in den Regressionsanalysen

\begin{tabular}{lr}
\hline Geschlecht weiblich & $56,5 \%$ \\
Geschlecht männlich (Referenz) & $43,5 \%$ \\
Befragter: kein Abitur (Referenz) & $33,9 \%$ \\
Befragter: Abitur & $28,7 \%$ \\
Befragter: (Fach-)Hochschulabschluss & $37,4 \%$ \\
Partner: kein (Fach-)Hochschulabschluss (Referenz) & $67,1 \%$ \\
Partner: (Fach-)Hochschulabschluss & $32,9 \%$ \\
Befragter: Leitende berufliche Position (Leitender Angestellter/leitender Beamter/selbstständig) & $24,4 \%$ \\
Befragter: keine leitende berufliche Position (Referenz) & $75,6 \%$ \\
Partner: Leitende berufliche Position & $25,2 \%$ \\
Partner: Keine leitende berufliche Position (Referenz) & $74,8 \%$ \\
Haushalt ohne Kinder (nach Umzug) (Referenz) & $72,8 \%$ \\
Haushalt mit Kindern (nach Umzug) & $27,2 \%$ \\
Par/Familie (nach Umzug) (Referenz) & $74,9 \%$ \\
Single/Alleinerziehend (nach Umzug) & $25,1 \%$ \\
Wohnort kein Oberzentrum (Referenz) & $70,7 \%$ \\
Wohnort Oberzentrum (Dortmund, Bochum, Essen, Wuppertal) & $29,3 \%$ \\
Distanz von Hagen Stadtmitte <20 km (Referenz) & $71,8 \%$ \\
Distanz von Hagen Stadtmitte 20-30 km & $19,2 \%$ \\
Distanz von Hagen Stadtmitte>30 km & $9,0 \%$ \\
Beruflicher Umzugsgrund hat keine Rolle gespielt (Referenz) & $55,2 \%$ \\
Beruflicher Umzugsgrund (hat auch eine Rolle gespielt oder ausschlaggebend) & $44,8 \%$
\end{tabular}

Die Werte in den Tabellen 9-10 weichen teilweise von den deskriptiven Ergebnisdarstellungen ab, weil in die Regressionsmodelle nur Datensätze mit vollständigen Informationen eingehen.

\section{Literatur}

Becker, G. S. (1981): A Treatise on the Family. Cambridge, London.

Blotevogel, H. H.; Münter, A.; Terfrüchte, T. (2009): Raumwissenschaftliche Studie zur Gliederung des Landes Nordrhein-Westfalen in regionale Kooperationsräume. Abschlussbericht. Dortmund.

Boarnet, M. G. (2011): A broader context for land use and travel behavior, and a research agenda. In: Journal of the American Planning Association 77, 3, 197-213.

Camstra, R. (1996): Commuting and gender in a lifestyle perspective. In: Urban Studies 33, 2, 283-300.

Clark, W. A. V.; Wang, W. W. (2005): Job access and commute penalties: balancing work and residence in Los Angeles. In: Urban Geography 26, 7, 610-626.
Cooke, T. J.; Boyle, P.; Couch, K.; Feijten, P. (2009): A longitudinal analysis of family migration and the gender gap in earnings in the United States and Great Britain. In: Demography 46, 1, 147-167.

Crane, R. (2007): Is there a quiet revolution in women's travel? Revisiting the gender gap in commuting. In: Journal of the American Planning Association 73, 3, 298-316.

Duncan, R. P.; Perrucci, C. C. (1976): Dual occupation families and migration. In: American Sociological Review 41, 2, 252-261.

Einig, K.; Jonas, A.; Zaspel, B. (2009): Eignung von CORINEGeodaten und Daten der Flächenerhebung zur Analyse der Siedlungs- und Verkehrsflächenentwicklung in Deutschland. Göttingen. $=$ Land Use Economics and Planning - Discussion Paper 2009-08 der Universität Göttingen. 
Frank, S. (2011): Je näher man hinschaut, desto fremder schaut es zurück. Aktuelle Diskussionen um Suburbanisierung und Gentrifizierung. In: Herrmann, H.; Keller, C.; Neef, R.; Ruhne, R. (Hrsg.): Die Besonderheit des Städtischen. Wiesbaden, 285-300.

Geier, S.; Holz-Rau, C.; Krafft-Neuhäuser, H. (2001): Randwanderung und Verkehr. In: Internationales Verkehrswesen 53, 1-2, 22-26.

Gordon, P.; Kumar, A.; Richardson, H. W. (1989): Gender differences in metropolitan travel behaviour. In: Regional Studies 23, 6, 499-510.

Green, A. E. (1997): A question of compromise? Case study evidence on the location and mobility strategies of dual career households. In: Regional Studies 31, 7, 641-657.

Grunow, D.; Schulz, F.; Blossfeld, H.-P. (2012): What determines change in the division of housework over the course of marriage? In: International Sociology 27, 3, 289-307.

Guth, D. (2014): Berufspendlerverkehr im Kontext (post)suburbaner Raumentwicklung: Trends seit 1970 (Dissertation, TU Dortmund). Dortmund.

Hakim, C. (2000): Work-Lifestyle Choices in the 21st Century: Preference Theory. Oxford.

Hanson, S.; Pratt, G. (1995): Gender, Work and Space. London.

Harris, C. D.; Ullman, E. L. (1945): The nature of cities. In: Annals of the American Academy of Political and Social Science 242, 7-17.

Hesse, M. (2001): Mobilität und Verkehr im suburbanen Kontext. In: Brake, K.; Dangschat, J. S.; Herfert, G. (Hrsg.): Suburbanisierung in Deutschland. Opladen, 97-108.

Hjorthol, R. (2008): Daily mobility of men and women - a barometer of gender equality? In: Uteng, T. P.; Cresswell, T. (2008): Gendered Mobilities. Aldershot, 193-210.

Hjorthol, R.; Vagane, L. (2014): Allocation of tasks, arrangement of working hours and commuting in different Norwegian households. In: Journal of Transport Geography 35, 75-83.

Hudson, C. (2014): Left holding the baby or increased career opportunities? The gendered consequences of regional enlargement and increased commuting. In: Women's Issues in Transportation. Proceedings of the 5th International Conference, Paris, 14-16 April 2014. Paris, 573-586.

Infas/DLR - Institut für angewandte Sozialwissenschaft $\mathrm{GmbH}$; Deutsches Zentrum für Luft- und Raumfahrt e. V. (2010): Mobilität in Deutschland 2008: Methodenbericht. Bonn, Berlin.

Kasper, B.; Scheiner, J. (2006): Räumliche Mobilität als Prozess kurzund langfristigen Handelns: Zusammenhänge zwischen Wohnund Alltagsmobilität. In: Beckmann, K. J.; Hesse, M.; Holz-Rau, C.; Hunecke, M. (Hrsg.): StadtLeben - Wohnen, Mobilität und Lebensstil. Wiesbaden, 167-186.

Kawase, M. (2004): Changing gender differences in commuting in the Tokyo metropolitan suburbs. In: GeoJournal 61, 3, 247-253.

Kloosterman, R. C.; Musterd, S. (2001): The Polycentric Urban Region: Towards a Research Agenda. In: Urban Studies 38, 4, 623-633.

König, K. (1978): Suburbanisierung und Stadtverkehr. Räumliche Differenzierung der Daseinsgrundfunktionen und deren Einflüsse auf den Pendelverkehr zur Kernstadt, dargestellt am Beispiel der Stadtregion Augsburg. In: Akademie für Raumforschung und Landesplanung (Hrsg.): Beiträge zum Problem der Suburbanisierung (Teil 2). Ziele und Instrumente der Planung im suburbanen Raum. Hannover, 127-156. = Forschungs- und Sitzungsberichte, 125.

Konrad, K. (2015): Wandel des Geschlechterverhältnisses - Wandel der Mobilität? Wiesbaden.

Krajewski, C.; Werring, J. (2013): Ländliche Wohnungsmärkte unter Schrumpfungsbedingungen. In: Standort - Zeitschrift für Angewandte Geographie 37, 2, 97-105.
LEG Immobilien AG (2014): LEG-Wohnungsmarktreport NRW 2014: Hagen. Düsseldorf.

MacDonald, H. I. (1999): Women's employment and commuting: Explaining the links. In: Journal of Planning Literature 13, 3, 267-283.

Matthies, E.; Kuhn, S.; Klöckner, C. A. (2002): Travel Mode Choice of Women: The Result of Limitation, Ecological Norm or Weak Habit? In: Environment and Behavior 34, 2, 163-177.

McQuaid, R. W.; Chen, T. (2012): Commuting times - The role of gender, children and part-time work. In: Research in Transportation Economics 34, 1, 66-73.

Nisic, N. (2010): Mitgegangen - mitgefangen? Die Folgen von Haushaltsumzügen für die Einkommenssituation von Frauen in Partnerschaften. In: Kölner Zeitschrift für Soziologie und Sozialpsychologie 62, 3, 515-549.

Prillwitz, J.; Harms, S.; Lanzendorf, M. (2007): Interactions between Residential Relocations, Life Course Events, and Daily Commute Distances. In: Transportation Research Record 2021, 64-69.

Rahn, C. (2011): Restriktionen und Optionen in Suburbia Genderspezifika von Arbeit, Mobilität und Sozialkapital im Berliner Umland (Dissertation an der Humboldt-Universität zu Berlin). Berlin.

Scheiner, J. (2009): Sozialer Wandel, Raum und Mobilität. Empirische Untersuchungen zur Subjektivierung der Verkehrsnachfrage. Wiesbaden.

Scheiner, J.; Holz-Rau, C. (2012): Gendered travel mode choice: a focus on car deficient households. In: Journal of Transport Geography 24, 250-261.

Schirbaum, A. (2014): Regionale Beschäftigungsstruktur in Nordrhein-Westfalen seit 2008. In: IT.NRW - Information und Technik Nordrhein-Westfalen (Hrsg.): Entwicklungen am Arbeitsmarkt Nordrhein-Westfalens. Düsseldorf, 4-13. = Statistische Analysen und Studien, 80.

Schmidt, S. (2014): Wanderungsmotivanalyse der Hagener StadtUmland-Wanderer. Unveröffentlichte Masterarbeit, TU Dortmund, Fakultät Raumplanung.

Schneider, N. F.; Limmer, R.; Ruckdeschel, K. (2002): Berufsmobilität und Lebensform. Sind berufliche Mobilitätserfordernisse in Zeiten der Globalisierung noch mit der Familie vereinbar? Stuttgart. = Schriftenreihe des Bundesministeriums für Familie, Senioren, Frauen und Jugend, 208.

Shearmur, R. (2006): Travel from Home: An Economic Geography of Commuting Distances in Montreal. In: Urban Geography 27, 4, 330-359.

Siedentop, S.; Hesse, M. (2005): Mobilität im suburbanen Raum. Neue verkehrliche und raumordnerische Implikationen des räumlichen Strukturwandels. Abschlussbericht, Teil A: Grundlagen. Dresden, Berlin/Erkner, Leipzig.

Sultana, S. (2005): Effects of married-couple dual-earner households on metropolitan commuting: evidence from the Atlanta metropolitan area. In: Urban Geography 26, 4, 328-352.

Surprenant-Legault, J.; Patterson, Z.; El-Geneidy, A. M. (2013): Commuting trade-offs and distance reduction in two-worker households. In: Transportation Research Part A 51, 12-28.

Walby, S. (1990): Theorizing Patriarchy. London.

Zolnik, E. J. (2011): The effects of sprawl on private-vehicle commuting distances and times. In: Environment and Planning B $38,6,1071-1084$. 